\title{
Phase diffusion of a two-component Bose-Einstein condensates: exact and short-time solutions for arbitrary coherent spin state
}

\author{
G. R. Jin, B. B. Wang, and Y. W. Lu \\ Department of Physics, Beijing Jiaotong University, Beijing 100044, China
}

\begin{abstract}
We investigate phase diffusion of a two-component Bose-Einstein condensates prepared initially in arbitrary coherent spin state $\left|\theta_{0}, \phi_{0}\right\rangle$. Analytical expression of the phase-diffusion time is presented for $\theta_{0} \neq \pi / 2$ case. In comparison with the symmetrical case (i.e., $\theta_{0}=\pi / 2$ ), we find that the diffusion process becomes slowly due to the reduced atom number variance.
\end{abstract}

PACS numbers: 03.75.Mn, 05.30.Jp,42.50.Lc

Phase diffusion of Bose-Einstein condensates (BECs) [1 7], aroused from atom-atom interactions destroys phase coherence, and thus sets a limit to the applications of the condensates in high-precision measurement and quantum information processing. The phase diffusion is observable by measuring fringe visibility in atomic interference experiments [8 12]. For a two-component BECs prepared initially in a coherent spin state (CSS) $\left|\theta_{0}, \phi_{0}\right\rangle$ with $\theta_{0}=\pi / 2$ [see Ref. [13], or Eq. (2)], it has been shown that the single-particle coherence (or fringe visibility) decays exponentially with the time scale $\chi t_{d}=(N / 2)^{-1 / 2}[2$ 5], dependent upon atom number $N$ and the self-interaction strength $\chi \sim\left(a_{11}+a_{22}-2 a_{12}\right) / 2$. Here, $a_{i i}$ and $a_{i j}(i \neq j)$ denote intra- and inter-species s-wave scattering lengths, respectively. In this paper, we generalize previous works by considering arbitrary initial CSS, i.e., $\theta_{0} \neq \pi / 2$. Exact and short-time solutions of the single-particle coherence are obtained. In comparison with $\theta_{0}=\pi / 2$ case, we find that the diffusion becomes slowly due to relatively narrow atom number variance.

Considering a two-component BECs with internal states $|1\rangle$ and $|2\rangle$ confined in a deep potential, we adopt single-mode approximation (SMA) [14], i.e., keeping the condensed-mode wave function $\Phi_{0}(r)$ for the two components, so the total system can be well described by second-quantized Hamiltonian $(\hbar=1)$ :

$$
\begin{aligned}
\hat{H} & =\omega_{1} \hat{n}_{1}+\omega_{2} \hat{n}_{2}+u_{12} \hat{n}_{1} \hat{n}_{2} \\
& +\frac{u_{11}}{2}\left(\hat{b}_{1}^{\dagger}\right)^{2}\left(\hat{b}_{1}\right)^{2}+\frac{u_{22}}{2}\left(\hat{b}_{2}^{\dagger}\right)^{2}\left(\hat{b}_{2}\right)^{2},
\end{aligned}
$$

where $\hat{b}_{i}, \hat{b}_{i}^{\dagger}$, and $\hat{n}_{i}\left(=\hat{b}_{i}^{\dagger} \hat{b}_{i}\right)$ are the annihilation, creation, and number operators for the internal states $i=$ 1,2 , respectively. In addition, $\omega_{i}$ are single-particle energies, and $u_{i j}=\left(4 \pi a_{i j} / M\right) \int d^{3} r\left|\Phi_{0}(r)\right|^{4}$ are atom-atom interaction strengthes. Particle number operator $\hat{N}=$ $\hat{n}_{1}+\hat{n}_{2}$ is a conserved quantity and is set to the c number $N$. Hamiltonian (1) can be rewritten as $\hat{H}=\delta \hat{J}_{z}+\chi \hat{J}_{z}^{2}$, where the detuning $\delta=\omega_{2}-\omega_{1}+\left(u_{22}-u_{11}\right)(N-1) / 2$, and the self-interaction strength $\chi=\left(u_{11}+u_{22}-2 u_{12}\right) / 2$. The spin operators $\hat{J}_{+}=\left(\hat{J}_{-}\right)^{\dagger}=\hat{b}_{2}^{\dagger} \hat{b}_{1}$ and $\hat{J}_{z}=\left(\hat{n}_{2}-\hat{n}_{1}\right) / 2$, obeying SU(2) algebra. The nonlinearity $\chi \hat{J}_{z}^{2}$ has been proposed to prepare spin squeezed state [15 17] and also quantum entangled state [18, 19]. Note that the model considered here with nonzero $\delta$ can be also used to study the BECs in an asymmetric double-well [20]. Squeezing via coupling of the BECs in a double-well potential with a cavity light field has been investigated in Ref. [21].

Besides the spin squeezing, mean-field interaction $\chi J_{z}^{2}$ also leads to phase diffusion in the two-component BECs [1 7, 12], which can be illuminated schematically by Husimi $Q$ function $Q(\theta, \phi ; t)=|\langle\theta, \phi \mid \Psi(t)\rangle|^{2}$, where

$$
|\theta, \phi\rangle=\exp \left\{i \theta\left(\hat{J}_{x} \sin \phi-\hat{J}_{y} \cos \phi\right)\right\}|j, j\rangle
$$

is arbitrary CSS [13] and $|\Psi(t)\rangle=e^{-i \hat{H} t}|\Psi(0)\rangle$ is a state vector at any time $t$. For an initial state $|\Psi(0)\rangle=\left|\theta_{0}, \phi_{0}\right\rangle$, analytic expression of the $Q$ function reads [13]

$$
Q(\theta, \phi ; 0)=\left|\left\langle\theta, \phi \mid \theta_{0}, \phi_{0}\right\rangle\right|^{2}=\left[\frac{1+\cos \Theta}{2}\right]^{2 j},
$$

where $\cos \Theta=\cos \theta \cos \theta_{0}+\sin \theta \sin \theta_{0} \cos \left(\phi-\phi_{0}\right)$. The $Q$ function can be plotted in three-dimensional phase space (i.e., Bloch sphere) [15, 16], or alternatively, in a two-dimensional phase space $\left(\phi, s_{z}\right)$ via a mapping $s_{z}=j \cos \theta[12,17,22]$. As shown in Fig. 1(a) and Fig. 1(b), the density of $Q(\theta, \phi ; 0)$ is distributed isotropically, indicating the minimal uncertainty relationship of the initial CSS. Under the government of nonlinear interaction $\chi J_{z}^{2}$, the system will evolve into a spin squeezed state 15. with anisotropic distribution of the $Q$ function [see Fig. [(c)]. As time increases, the spin system is oversqueezed [15, 23] and shows a spread of the $Q$ function along the $\phi$ axis, which simulates an increased relative phase fluctuation (i.e., phase diffusion) [12], as shown in Fig. 1(d).

It was shown that the most sensitive states to the diffusion are the symmetrical CSS with $\theta_{0}=\pi / 2$, corresponding to equal populations between the two internal states [7]. The phase-diffusion time scale is $\chi t_{d}=1 / \sqrt{j}$ (with $j=N / 2$ ) $2[5]$. Here, we generalize it for arbitrary initial CSS

$$
|\Psi(0)\rangle=\left|\theta_{0}, \phi_{0}\right\rangle=\sum_{m=-j}^{j} c_{m}|j, m\rangle,
$$




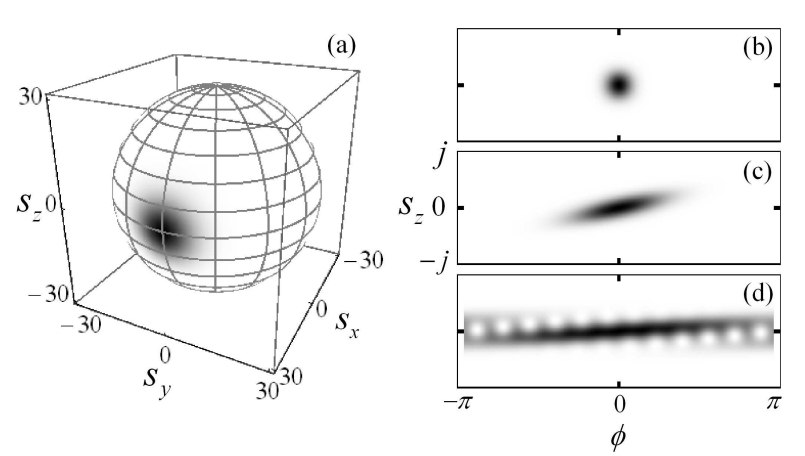

FIG. 1: Husimi $Q$ function $Q(\theta, \phi ; t)$ for initial $\operatorname{CSS} \mid \theta_{0}=$ $\left.\pi / 2, \phi_{0}=0\right\rangle$ on Bloch sphere (a), with Bloch vector $\vec{s}=$ $\langle\vec{J}\rangle=j(\sin \theta \cos \phi, \sin \theta \sin \phi, \cos \theta)$ and $j=N / 2=30$. The $Q$ functions in the phase space $\left(\phi, s_{z}\right)$ for various times: (b) $t=0$, (c) $t=t_{s}=0.0764 \chi^{-1}$, and (d) $t=2 t_{d}=0.2651 \chi^{-1}$. Time scale $\chi t_{s} \simeq 3^{1 / 6}(2 j)^{-2 / 3}$ is for optimal spin squeezing [15-17], and $\chi t_{d}=j^{-1 / 2}$ is phase-diffusion time [2-5]. The $Q$ function is normalized by its maximum value.

where the amplitudes

$$
c_{m}=\left(\begin{array}{c}
2 j \\
j-m
\end{array}\right)^{1 / 2} \cos ^{j+m}\left(\frac{\theta_{0}}{2}\right) \sin ^{j-m}\left(\frac{\theta_{0}}{2}\right) e^{i(j-m) \phi_{0}(.5)}
$$

In single-particle picture, the CSS corresponds to all the atoms occupying a superposed state [15]: $\cos \left(\theta_{0} / 2\right)|2\rangle+$ $e^{i \phi_{0}} \sin \left(\theta_{0} / 2\right)|1\rangle$, where the polar angle $\theta_{0}$ and the azimuth angle $\phi_{0}$ determine atom population and the relative phase between the two internal states, respectively. In Fig. 2, we plot $\left|c_{m}\right|^{2}$ as a function of $m$. One can find that the probability distribution of the CSS can be treated as a Gaussian wave packet [see below Eq. (11)]. At any time $t$, the spin system evolves into

$$
|\Psi(t)\rangle=\sum_{m=-j}^{j} c_{m} e^{-i\left(\delta m+\chi m^{2}\right) t}|j, m\rangle
$$

i.e., a superposition of atomic number state $|j, m\rangle=$ $|j-m\rangle_{1}|j+m\rangle_{2}$. Due to the presence of atom-atom interaction $(\chi \neq 0)$, each number state has different phase evolution rate, which in turn lead to collapse and revival of the Rabi oscillation, a phenomenon that is wellknown in quantum optics [14]. Similar effect has been investigated in two-component BECs 24], atom-molecular BECs [25 27], and exciton emission [28 30].

Phase diffusion of the BECs considered here is in fact collapse of the first-order temporal correlation function [3, 7]:

$$
g_{12}^{(1)}=\frac{\left|\rho_{12}^{(1)}\right|}{\sqrt{\rho_{11}^{(1)} \rho_{22}^{(1)}}} \equiv \frac{\left|\left\langle\hat{J}_{+}\right\rangle\right|}{\sqrt{j^{2}-\left\langle\hat{J}_{z}\right\rangle^{2}}}
$$

where $\rho_{k l}^{(1)}=\left\langle\hat{b}_{k}^{\dagger} \hat{b}_{l}\right\rangle / N$ with $k, l=1,2$ are the elements of the single-particle density matrix [7]. The coherence $g_{12}^{(1)}$ is observable in experiments by extracting the visibility of the interference fringes [8 12]. The expectation value $\left\langle\hat{J}_{z}\right\rangle=\left\langle\Psi(t)\left|\hat{J}_{z}\right| \Psi(t)\right\rangle$, reads

$$
\left\langle\hat{J}_{z}\right\rangle=\sum_{m=-j}^{j} m\left|c_{m}\right|^{2}=j \cos \theta_{0}
$$

which is a constant for a fixed polar angle of the initial $\operatorname{CSS} \theta_{0}$. This is because of conserved operator $\hat{J}_{z}$ with respect to the Hamiltonian $\hat{H}$. Similarly, we obtain the expectation value $\left\langle\hat{J}_{z}^{2}\right\rangle=j^{2} \cos ^{2} \theta_{0}+(j / 2) \sin ^{2} \theta_{0}$, and

$$
\begin{aligned}
\left\langle\hat{J}_{+}\right\rangle & =e^{i\left(\phi_{0}+\delta t+\chi t\right)} \cot \frac{\theta_{0}}{2} \sum_{m=-j}^{j}(j-m)\left|c_{m}\right|^{2} e^{2 i m \chi t} \\
& =j e^{i\left(\phi_{0}+\delta t\right)} \sin \theta_{0}\left[\cos \chi t+i \cos \theta_{0} \sin \chi t\right]^{2 j-1}(9)
\end{aligned}
$$

where we have used the relation: $c_{m+1}=(j-m)^{1 / 2}(j+$ $m+1)^{-1 / 2} \cot \left(\theta_{0} / 2\right) e^{-i \phi_{0}} c_{m}$. Inserting Eq. (8) and Eq. (9) into Eq. (7), we further obtain the exact solution of the coherence

$$
g_{12}^{(1)}(t)=\left[1-\sin ^{2}\left(\theta_{0}\right) \sin ^{2}(\chi t)\right]^{j-1 / 2},
$$

which shows a decay of the coherence, i.e., phase diffusion. Such a dephasing process depends sensitively on the self-interaction strength $\chi$ and the polar angle $\theta_{0}$ of the initial CSS; while the detuning $\delta$ and the azimuth angle $\phi_{0}$ gives vanishing contribution to the coherence.
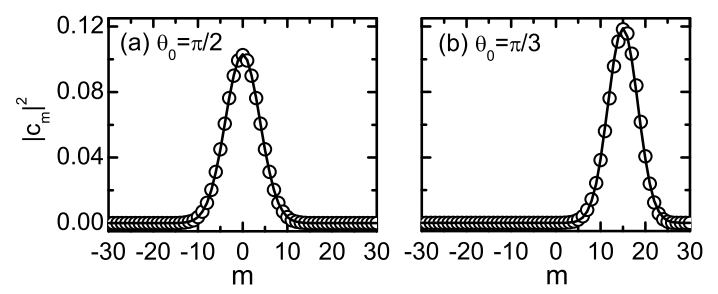

FIG. 2: Probability distribution, $\left|c_{m}\right|^{2}$ as a function of $m$ for the initial CSS $\left|\theta_{0}, \phi_{0}\right\rangle$. (a) $\theta_{0}=\pi / 2$ and (b) $\theta_{0}=\pi / 3$. The empty circle is given by Eq. (5), and solid line is given by Eq. (11). The parameter $j=N / 2=30$.

Time scale of the diffusion is determined by short-time behavior of the coherence. Following Imamoğlu et al. [3], we treat the initial CSS as a Guassian wave-packet with its peak located at $m \simeq\left\langle\hat{J}_{z}\right\rangle=j \cos \theta_{0}$, namely

$$
\left|c_{m}\right|^{2} \simeq \frac{1}{\left[2 \pi\left(\Delta \hat{J}_{z}\right)^{2}\right]^{1 / 2}} \exp \left[-\frac{\left(m-\left\langle\hat{J}_{z}\right\rangle\right)^{2}}{2\left(\Delta \hat{J}_{z}\right)^{2}}\right]
$$

where the width

$$
\left(\Delta \hat{J}_{z}\right)^{2}=\left\langle\hat{J}_{z}^{2}\right\rangle-\left\langle\hat{J}_{z}\right\rangle^{2}=(j / 2) \sin ^{2} \theta_{0}
$$

Due to conserved particle number $N(=2 j), \hat{n}_{1}=j-$ $\hat{J}_{z}$ and $\hat{n}_{2}=j+\hat{J}_{z}$, and thus atom number variances $\left(\Delta \hat{n}_{1}\right)^{2}=\left(\Delta \hat{n}_{2}\right)^{2}=\left(\Delta \hat{J}_{z}\right)^{2}[17]$. 
In Fig. 2. we check the validity of Eq. (11) by comparing it with the exact result of $\left|c_{m}\right|^{2}$ [where $c_{m}$ is given by Eq. (5)], and find both results fit with each other. For symmetric BEC with $\theta_{0}=\pi / 2$, we have $\left\langle\hat{J}_{z}\right\rangle=0$ and atom number variance $\left(\Delta \hat{J}_{z}\right)^{2}=j / 2$; while for $\theta_{0} \neq \pi / 2$, the variance (width) becomes narrow, as shown in Fig. 2(b). Inserting Eq. (11) into Eq. (9), and replacing the discrete sum over $m$ by an integral, we get

$$
\begin{aligned}
\left\langle\hat{J}_{+}\right\rangle \simeq & \frac{1}{\sqrt{\pi}} e^{i\left(\phi_{0}+\delta t+\chi t\right)} e^{2 i\left\langle\hat{J}_{z}\right\rangle \chi t} \cot \frac{\theta_{0}}{2} \\
& \times \int_{\rho_{\min }}^{\rho_{\max }}\left[j-\left\langle\hat{J}_{z}\right\rangle-\sqrt{2}\left(\Delta \hat{J}_{z}\right) \rho\right] \\
& \times \exp \left[-\rho^{2}+2 i \sqrt{2} \chi t\left(\Delta \hat{J}_{z}\right) \rho\right] d \rho,
\end{aligned}
$$

where we have set

$$
\rho=\frac{m-\left\langle\hat{J}_{z}\right\rangle}{\sqrt{2}\left(\Delta \hat{J}_{z}\right)} .
$$

For $\theta_{0} \sim \pi / 2$ and $j \rightarrow \infty$ (large $N$ limit), the integral upper limit $\rho_{\max }=\sqrt{j} \tan \left(\theta_{0} / 2\right) \rightarrow \infty$ and the lower limit $\rho_{\text {min }}=-\sqrt{j} \cot \left(\theta_{0} / 2\right) \rightarrow-\infty$. In the short-time limit $(\chi t \ll 1), 1-2 i \chi t \cos ^{2}\left(\theta_{0} / 2\right) \simeq \exp \left\{-2 i \chi t \cos ^{2}\left(\theta_{0} / 2\right)\right\}$, and thus from Eq. (13) we obtain

$$
\begin{aligned}
\left\langle\hat{J}_{+}\right\rangle \simeq & j \sin \left(\theta_{0}\right) \exp \left\{i\left[\phi_{0}+\delta t+\chi t(2 j-1) \cos \theta_{0}\right]\right\} \\
& \times \exp \left[-2 \chi^{2}\left(\Delta \hat{J}_{z}\right)^{2} t^{2}\right],
\end{aligned}
$$

where two integrals: $\int_{-\infty}^{\infty} e^{-\rho^{2}} e^{i \lambda \rho} d \rho=\sqrt{\pi} e^{-\lambda^{2} / 4}$ and $\int_{-\infty}^{\infty} \rho e^{-\rho^{2}} e^{i \lambda \rho} d \rho=i \lambda \sqrt{\pi} e^{-\lambda^{2} / 4} / 2$ have been used in derive of the above result. Thus, the short-time solution of the coherence reads

$$
g_{12}^{(1)}(t) \simeq \exp \left[-2 \chi^{2}\left(\Delta \hat{J}_{z}\right)^{2} t^{2}\right] \equiv \exp \left[-\left(t / t_{d}\right)^{2}\right],
$$

with characteristic time scale of the phase diffusion

$$
\chi t_{d}=\frac{1}{\sqrt{2}\left(\Delta \hat{J}_{z}\right)}=\frac{1}{\sqrt{j} \sin \theta_{0}} .
$$

Obviously, the phase-diffusion time $\chi t_{d}=1 / \sqrt{j}$ for the initial CSS with $\theta_{0}=\pi / 2\left[3,7\right.$, 12]; while for $\theta_{0} \neq \pi / 2$, our results show that the diffusion becomes slowly due to an enhanced factor $\sin \theta_{0}$ in the phase-diffusion rate [see also Fig. 3. The revival of the coherence occurring later at a time $\chi t_{r}=\pi[5,7]$ is observable in real experiment, such as $t_{r}=\pi / \chi \simeq 108.7$ microsecond for nonlinearity $\chi \simeq 2 \pi \times 4.6 \mathrm{~Hz}$ and particle number $N=60$ [12].

Finally, we note that similar results of Eq. (9) and Eq. (15) have been obtained in Ref. [31]. However, the authors focused on the increase of the diffusion time as $\chi \rightarrow 0$, but not $\theta_{0} \neq \pi / 2$. In addition, we emphasis that for negative $\chi$ the so-called phase separation may take place due to dynamically unstable of the BECs 32 39]. Both the phase diffusion and the phase separation

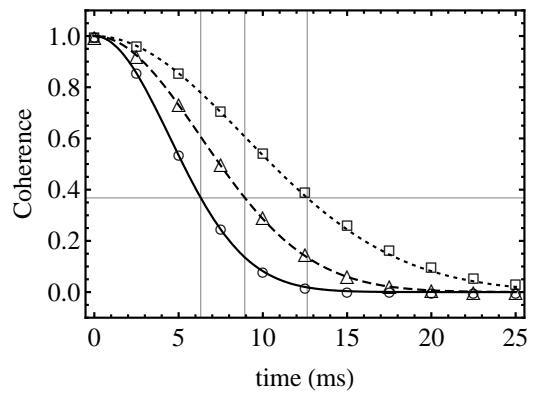

FIG. 3: Time evolution of the first-order temporal coherence $g_{12}^{(1)}(t)$ for various polar angles: $\theta_{0}=\pi / 6$ (dotted line, squares), $\pi / 4$ (dashed line, triangles), and $\pi / 2$ (solid line, circles). The empty squares, triangles, and circles are given by Eq. (10) for corresponding $\theta_{0}$ 's, while the curves are given by Eq. (16). Horizontal grid line gives the value 1/e, vertical lines denote the diffusion times $t_{d}$ for different $\theta$ 's. Other parameters are $N=60, \chi=2 \pi \times 4.6 \mathrm{~Hz}[12]$.

reduce the Ramsey fringes' visibility (i.e., the first-order coherence $\left.g_{12}^{(1)}\right)$. However, the latter effect results from dynamics of spatial degree-of-freedom, rather than that of the internal states considered here.

In summary, we have investigated phase diffusion of two-component BEC for arbitrary initial CSS. We show analytically that for the CSS with $\theta_{0} \neq \pi / 2$, the diffusion process is suppressed due to the reduced atom number variance $\left(\Delta \hat{J}_{z}\right)^{2}$ below the standard quantum limit $j / 2$ [see also Eqs. (12) and (17)]. Our analytic results are based upon the SMA, which works well to describe the condensates tightly confined in a three-dimensional (3D) trapping potential. For a lower dimensional cases [40], the coherence $g_{12}^{(1)}$ [or the fringe visibility] is expected to decay more quickly due to the whole continuum of excitation modes in confined degrees of freedom [12, 41].

\section{Acknowledgments}

This work is supported by the NSFC (Contract No. 10804007).

[1] Lewenstein M and You L 1996 Phys. Rev. Lett. 773489

[2] Wright E M, Walls D F, and Garrison J C 1996 Phys. Rev. Lett. 772158

[3] Imamōglu A, Lewenstein M, and You L 1997 Phys. Rev. Lett. 782511.

[4] Javanainen J and Wilkens M 1997 Phys. Rev. Lett. 78 4675; Javanainen J and Ivanov M. Y. 1999 Phys. Rev. A 602351

[5] Castin Y and Dalibard J 1997 Phys. Rev. A 554330

[6] Law C K, Pu H, Bigelow N P, and Eberly J H 1998 Phys. Rev. A $\mathbf{5 8} 531$ 
[7] Khodorkovsky Y, Kurizki G, and Vardi A 2008 Phys. Rev. Lett. 100220403

[8] Orzel C, Tuchman A K, Fenselau M L, Yasuda M, and Kasevich M A 2001 Science 291 2386;

Greiner M, Mandel O, Esslinger T, Hänsch T W, Bloch I 2002 Nature 415 39;

Li W, Tuchman A K, Chien H-C, and Kasevich M A 2007 Phys. Rev. Lett. 98040402

[9] Schumm T, Hofferberth S, Andersson L M, Wildermuth S, Groth S, Bar-Joseph I, Schmiedmayer J, Krüger P 2005 Nature Phys. 1 57;

Shin Y, Sanner C, Jo G-B, Pasquini T A, Saba M, Ketterle W, Pritchard D E, Vengalattore M, and Prentiss M 2005 Phys. Rev. A 72 021604(R)

[10] Chuu C-S, Schreck F, Meyrath T P, Hanssen J L, Price G N, and Raizen M G 2005 Phys. Rev. Lett. 95260403

[11] Jo G-B, Shin Y, Will S, Pasquini T A, Saba M, Ketterle W, Pritchard D E, Vengalattore M, and Prentiss M 2007 Phys. Rev. Lett. 98030407

[12] Widera A, Trotzky S, Cheinet P, Fölling S, Gerbier F, Bloch I, Gritsev V, Lukin M D, and Demler E 2008 Phys. Rev. Lett. 100140401

[13] Radcliffe J M 1971 J. Phys. A 4 313; Arecchi F T, Courtens E, Gilmore R, and Thomas H 1972 Phys. Rev. A 62211

[14] Walls D F and Milburn G J 2008 Quantum Optics (the 2nd edition, Springer-Verlag, Berlin Heidelberg), and references therein

[15] Kitagawa M and Ueda M 1993 Phys. Rev. A 47 5138; Sørensen A, Duan L M, Cirac J I, Zoller P 2001 Nature 40963

[16] Jin G R and Kim S W 2007 Phys. Rev. A 76 043621; ibid. 2007 Phys. Rev. Lett. 99170405

[17] Jin G R and Law C K 2008 Phys. Rev. A 78063620

[18] Wei W 2005 Chinese Physics A 142407

[19] Cheng R and Liang J-Q 2007 Chinese Physics A 16834

[20] Wang D-L, Wang F-J, Xiao Y-F and Yan X-H 2006, Acta Physica Sinica 55547

[21] Kong L-B, Zhan M-S, and Zhou L 2008, Chinese Physics B 171601

[22] Trimborn F, Witthaut D, and Korsch H J 2009 Phys. Rev. A 79013608

[23] Shalm L K, Adamson R B A, and Steinberg A M 2009 Nature 45767
[24] Kuang L M and Ouyang Z W 2000 Phys. Rev. A 61 023604; Kuang L M and Zhou L 2003 Phys. Rev. A 68 043606; Li W-D, Zhou X-J, Wang Y-Q, Liang J-Q, and Liu W-M 2001 Phys. Rev. A 64015602

[25] Javanainen J and Mackie M 1999, Phys. Rev. A 59, R3186; Vardi A, Yurovsky V A, and Anglin J R 2001, Phys. Rev. A 64063611

[26] Jin G R, Kim C K, and Nahm K 2005 Phys. Rev. A 72 045602

[27] Cai W, Jing H, Zhan M-S, Xu J-J 2007 Chin. Phys. Lett. 24867

[28] Jin G R and Liu W M 2004 Phys. Rev. A 70 013803; Jin G R, Liang Z X, and Liu W M 2004 J. Opt. B: Quantum Semiclass. Opt. 6296

[29] Glazov M M and Kavokin K V 2006 Phys. Rev. B 73 245317

[30] Li W, Song P, Liu J, Yang X 2007 J. Phys. B 401957

[31] Sinatra A and Castin Y 2000 Eur. Phys J. D 8319

[32] Ao P and Chui S T 1998 Phys. Rev. A 58 4836; Wu Biao and Niu Qian 2001, Phys. Rev. A 64 061603(R); Konotop V V and Salerno M 2002 Phys. Rev. A 65 021602(R)

[33] Kasamatsu K and Tsubota M 2004 Phys. Rev. Lett. 93 100402; Kasamatsu K and Tsubota M 2006 Phys. Rev. A 74013617

[34] Li L, Li Z, Malomed B A, Mihalache D, Liu W M 2005 Phys. Rev. A 72 033611; Jin G R, Kim C K, and Nahm K 2005 Phys. Rev. A 72045601

[35] Belmonte-Beitia J, Pérez-García V M, Vekslerchik V 2007 Chaos, Solitons and Fractals, 321268

[36] Huang Jin-Song, Chen Hai-Feng, and Xie Zhen-Wei 2008 Acta Phys. Sinica $\mathbf{5 7} 3435$

[37] Sorokinaa E A, Maimistova A I and Elyutin S O 2009 Physica D 2381394

[38] Alon O E, Streltsov A I, and Cederbaum L S 2009 Phys. Rev. A 79022503

[39] Baizakov B B, Bouketir A, Messikh A, and Umarov B A 2009 Phys. Rev. E 79046605

[40] Cui H-T, Wang L-C, and Yi X-X 2004 Acta Physica Sinica $\mathbf{5 3} 991$

[41] Bistritzer R and Altman E 2007 PNAS 104 9955; Burkov A A, Lukin M D, and Demler E 2007 Phys. Rev. Lett. 98200404. 